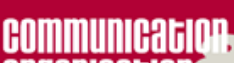

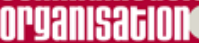

Communication et organisation

Revue scientifique francophone en Communication organisationnelle

$52 \mid 2017$

Communauté, sociabilité et bien commun: approche internationale

\title{
Les jeux des enfants dans le regard des adultes: appropriation et formation de communautés de jeu
}

Children's games at the adults view: appropriation and formation of game communities

Los juegos de niños en la mirada de los adultos: apropiación y formación de comunidades de juego

Os jogos infantis a partir do olhar dos adultos : apropriação e formação de comunidades de jogo

\section{Carolina Duek et Noelia Enriz}

\section{(2) OpenEdition}

\section{Journals}

Édition électronique

URL : https://journals.openedition.org/communicationorganisation/5679

DOI : 10.4000/communicationorganisation.5679

ISBN : 979-10-300-0257-7

ISSN : 1775-3546

\section{Éditeur}

Presses universitaires de Bordeaux

\section{Édition imprimée}

Date de publication : 1 décembre 2017

Pagination : 85-98

ISBN : 979-10-300-0163-1

ISSN : 1168-5549

Référence électronique

Carolina Duek et Noelia Enriz, «Les jeux des enfants dans le regard des adultes : appropriation et formation de communautés de jeu », Communication et organisation [En ligne], 52 | 2017, mis en ligne le 01 décembre 2020, consulté le 03 janvier 2022. URL : http://journals.openedition.org/ communicationorganisation/5679; DOI : https://doi.org/10.4000/communicationorganisation.5679 


\title{
Les jeux des enfants dans le regard des adultes: appropriation et formation de communautés de jeu
}

\author{
Corolina Duek ${ }^{1}$, Noelia Enriz $^{2}$
}

\section{Introduction}

Le jeu, d'après Huizinga (1938), est l'un des espaces dans lesquels se développe la culture. Il considère que l'homo ludens (l'homme qui joue) occupe sa place à côté de l'homo faber (op. cit). Or, le jeu suppose une improvisation libre (Callois 1967) et il peut être divisé en deux axes : d'un côté, la structure, et de l'autre, le contenu. La structure varie selon les sujets, mais le contenu est " fortement lié à la culture de leur époque, à leur appartenance sociale, à leur expérience et à leurs conditions de vie » (Malajovich et Windler 2000 : 119).

Dans cet article, nous analyserons le jeu comme le terrain où se cristallisent diverses formes d'appropriation de discours et de pratiques, aussi bien des moyens de communication que d'autres institutions qui désignent et qui utilisent le jeu selon différentes finalités dans l'espace complexe des parcours sociaux, culturels et éducatifs des sujets (Milstein et Mendes 1999 ; Orozco Gómez 1994 ; Mantilla 1996 ; De La Ville et Manson 2013). Nous étudierons simultanément deux espaces essentiels de la vie quotidienne: l'école et la maison. La communauté, formée par le collectif de joueurs (Enriz 2011), est le prélude de la communitas (Esposito 2007) au sein de laquelle le jeu prend un sens communautaire.

Nous examinerons le cas de la population Mbyá Guaraní à Misiones (Argentine) au sein de laquelle le jeu prend une importance toute particulière dans le temps où l'accès à l'école est limité. Le jeu, dans les communautés guaraníes, est une manière de s'approprier leur environnement: les pratiques ludiques des enfants sont, pour eux, une manière d'interpréter

1 Carolina Duek est chercheuse au CONICET et professeure à l'Université de Buenos Aires, Argentine. Elle a publié quatre ouvrages, notamment « Jeu, Jouets et novelles technologies » (Capital Intelectual). Elle fait sa recherche sur les enfants, les jeux, le genre et les médias. Elle est éditrice et directrice du journal académique «Lúdicamente "; duekcarolina@gmail.com

2 Noelia Enriz est chercheuse au CONICET et professeure à l'Université de Buenos Aires, Argentine. Elle a publié des articles dans des différents journaux académiques. Elle fait sa recherche sur des communautés guaranies à Misiones, Argentine. Elle est éditrice et directrice du journal académique « Lúdicamente »; noelia.enriz@gmail.com 
leur propre communauté et leur place dans les différents cercles familiaux et éducatifs (Enriz et Padawer 2009). Lécole n'occupe pas le centre de la vie communautaire en raison d'impossibilités structurelles (documentation nécessaire, distance avec les centres éducatifs). C'est la raison pour laquelle la notion de communautés de pratiques devient centrale pour comprendre le rôle des jeux dans l'éducation des enfants au sein de leur communauté (Lave et Wagner 2007). Les enfants avancent dans la compréhension du monde à la faveur d'activités créatives partagées avec d'autres enfants (Rogoff 1993).

$\mathrm{La}$ famille, produit de profondes mutations conçues tout au long des siècles, se consolide sous un modèle hégémonique de famille patriarcale pour les couches sociales bourgeoises (Habermas, 1981). La famille est un domaine où les enfants acquièrent un certain nombre de normes nécessaires pour vivre en société, mais, en même temps, c'est un refuge, un espace séparé des « lieux communs ", dans lequel on peut agir plus libre et spontanément. La fonction de la famille comme institution centrale pour comprendre la construction de l'enfance, se rapporte, pour Sennett (1978), à la garde de l'enfant, aux loisirs, et à la quotidienneté affective. Cette institution, aidée par les normes sociales acquises, permettrait (ou semblerait permettre) plus de liberté. Ce sera donc dans l'école, dans sa création, dans la définition de ses fonctions et à travers la discipline de classe que l'État complémentera la formation des enfants pour garantir leur « citoyenneté » afin qu'ils puissent s'insérer dans une société en tant qu'« adultes normalisés ». Dans ce cadre, l'éducation devient alors un facteur d'homogénéisation sociale et participe de la construction d'un récit sur l'égalité des individus (Alvarez Uría et Varela 1991).

Dans cet article, nous nous proposons d'identifier les formes dans lesquelles les contextes du jeu conditionnent les pratiques ludiques et de caractériser les appropriations que les sujets impliqués font de ceux-ci dans les domaines mentionnés. Nous soutiendrons, comme hypothèse, que le jeu est un espace dans lequel les enfants produisent et reproduisent des aspects de la vie sociale liés à leurs parcours culturels, éducatifs, économiques et sociaux. Et, qu'en plus, les appropriations « utilitaires » du jeu ne proviennent jamais des enfants (garçons ou filles) mais des adultes qui voient, dans cette interaction ludique, la possibilité d'en tirer bénéfice.

Le jeu utile sera la dimension transversale au texte, et les différents points s'organiseront tout autour de la relation entre le jeu, les nouvelles technologies de l'information et de la communication, les jeux traditionnels et le rapport avec les communautés qui se construisent autour des pratiques.

L'une des grandes difficultés rencontrées au cours de cette analyse, est liée à sa dimension subjective : si ce sont les joueurs qui décident, proposent et soutiennent les espaces ludiques, comment pourrait-on parler des jeux ? Nous avons donc enquêté auprès d'enfants des deux sexes ainsi qu'auprès de leurs parents afin d'identifier, à travers leurs propres mots, les formes et le sens qu'acquièrent les pratiques ludiques. Par ailleurs, en dépit de difficultés 
bureaucratiques pour accéder aux institutions scolaires, nous avons pu enquêter auprès des enseignants et des services administratifs de la ville de Buenos Aires. Toutes ces enquêtes constituent un corpus de travail sur lequel sera axé cet article.

\section{Le jeu et la communauté}

Le fait de jouer peut être interprété comme une faute si cela arrive au milieu d'une activité obligatoire, telle qu'une classe de Mathématiques à l'école. Le jeu que les enfants peuvent eux-mêmes concevoir comme une activité volontairement choisie se pratique bien souvent « en cachette » au milieu des obligations, pour échapper, en quelque sorte, à la réalité. Passer une " cocotte en papier » (aussi nommée " pouce-pouce » ou « cube magique ») de main en main, est une activité réalisée à l'insu du maître, sous les tables de la classe. Cette pratique qui cherche à contourner une éventuelle sanction, est donc une manière de soustraire à l'autorité du maître. L'objet ludique en question exige une certaine habileté manuelle pour le construire et en faciliter l'usage. À cela s'ajoutent le choix puis l'écriture des différents mots ou couleurs pour créer le répertoire qui s'inscrit sur ses faces internes. Dans la "cocotte en papier", on peut noter des actions, des prénoms, des gages, etc. Elle peut être utilisée pour deviner une couleur préférée, quelque chose qu'on peut faire, ou bien « le garçon qui te plaît ». La dynamique du jeu est la suivante: un garçon ou une fille choisit un chiffre, on fait bouger sur les quatre doigts la cocotte pour compter, puis on opte pour l'une des options disponibles. Jouer à la «cocotte en papier» ne se résume pas seulement à prendre une série de décisions : il faut que le lieu, le temps et la façon d'y jouer soient en tension face à l'autorité et les règles.

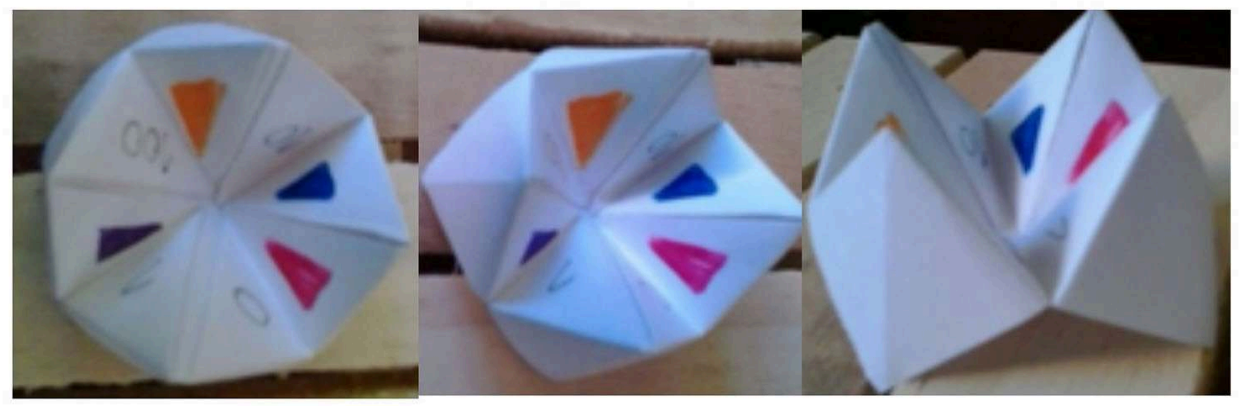

Image 1 : Set d'images d'une « cocotte en papier » (images des auteures)

Le jeu de la « cocotte en papier» implique un accord entre les membres, mais en même temps la possibilité d'une communitas (Espósito, 2007) dans la mesure où celle-ci les intègre dans un collectif d'obligations réciproques. Les enfants sont dans un procès de constitution subjective et c'est la raison 
pour laquelle ils nouent des liens significatifs tout en utilisant des objets qui organisent leurs pratiques. La communauté de jeu forme donc ce collectif au sein duquel les enfants apprennent à coopérer, à interagir, à décider selon les règles spécifiques du jeu dans le contexte de leur vie quotidienne. Le jeu devient donc, pour les enfants, dans un tel contexte, un facteur déterminant du lien social dans la mesure où il constitue pour eux une manière de préparer, d'anticiper leur insertion dans la communauté des adultes.

Le développement de cette pratique relève d'un processus communautaire : elle suppose l'exclusion d'autres sujets, nécessaire à la formation de toute communauté, qui, dans le cas présenté, pourrait se centrer sur l'enseignant. Tel que nous l'avons analysé dans d'autres cas (Enriz 2011) la figure d'une " communauté de jeu » permet d'identifier un groupe d'interpaires varié, qui partage des scènes de jeu dans différents espaces communs.

Autour du jeu se déploient des habiletés et des conditionnements dont les types sont très divers (Brougère 2013). La contribution des actions ludiques à la sociabilité des sujets devient l'un des éléments privilégiés au moment de faire l'éloge du jeu. Lorsqu'on pense à l'une des taxonomies la plus consolidée $\mathrm{du}$ domaine de l'investigation sur le jeu, celle de Roger Caillois (op. cit.), on retrouve que la sociabilité est conçue comme un facteur qui traverse toutes les dimensions du jeu. Caillois effectue une classification des jeux qu'il considère impulsée par une liberté initiale et par une nécessité de détente : agon, alea, mimicry et Ilynx constituent les quatre dimensions de cette classification. Ces jeux de compétition, de hasard, de représentation et imitation, et de vertige composent la taxonomie probablement la plus reprise qui organise le domaine du jeu dans le cadre des sciences sociales (Duek 2012 et Enriz 2011).

Nous pourrions dire de même à propos des approches de Lévi-Strauss (1966) relatives au jeu. Dans son analyse des systèmes classificatoires humains, il présente une analogie entre la fonction sociale des jeux et celle des rituels : tous les deux servent à intégrer des événements dans des structures. En outre, l'écart entre les deux n'est que le sens social qu'ils produisent : dans un cas (le rituel), il favorise l'affinité entre les termes ou groupes, alors que dans l'autre (le jeu), il rétablit les rivalités. S'il existait un dieu du jeu, avant de commencer une partie d'échecs, les deux joueurs partageraient leurs prières; plus tard, devant l'échiquier, gagner ou perdre en ferait des rivaux.

Poussé à l'extrême, évidemment, le jeu profond (Geertz 1972) est l'imbrication sociale d'une pratique. Et dans son développement, l'emphase est mise sur la portée de l'interdiction auprès de certains jeux. La communauté représente ce qui entoure les sujets et leur empêche d'être tels qu'ils sont puisqu'elle les précède et elle les excède (Espósito 2007).

\section{Le jeu dans l'école}

L'un des aspects des jeux nommés " traditionnels ", c'est d'avoir généré la sociabilité, dans la durée, chez des acteurs très divers. La catégorie de « jeux 
traditionnels » est habituellement attribuée à des pratiques de jeu dont on peut retrouver la trace tout au long du temps. Il n'existe pas de définition universelle ni de regard unique à propos des jeux traditionnels. On dirait plutôt que les divers groupes sociaux confèrent des valeurs différentielles à la tradition et aussi aux jeux qu'ils incluent dans cette catégorie-là en fonction des expériences et des parcours sociaux et historiques spécifiques. Le jeu traditionnel est, par définition, un objet résiduel de la culture contemporaine (Williams 1998) : conçu dans le passé et réaffirmé au présent comme une pratique actuelle, mais aussi, comme un fait résiduel d'un passé commun, significatif et structurant. La communauté impliquée dans cette pratique peut être abordée en termes de multiplicité et métamorphose (Gramaccia 2013) par le processus complexe de conformation que cela suppose quant à sa temporalité, son ampleur et ses hiérarchies.

Nous avons analysé, par exemple, l'utilisation du domino en Amérique Latine, avec des caractéristiques changeantes, sur une longue période (Duek et Enriz, 2015). Dans l'actualité, si un enfant est admis dans le jeu du domino, plusieurs scénarios sont possibles. On peut prendre l'exemple d'un domino qui incorpore des images du marché de la consommation ou le domino classique (avec des points). Dans les deux cas, les enfants développeront un jeu avec les autres tout en suivant les règles qui ordonnent les interactions (Chapela 2002 et Aizencang 2005). L'une des dimensions cruciales pour analyser les jeux, c'est le profil de ceux qui le proposent : ce n'est pas la même chose de jouer aux dominos de «Barbie » que de jouer à celui des lettres (enchaîner une pièce à une autre en identifiant leur forme) dans un contexte scolaire. Dans les deux cas, la dynamique et les règles du jeu sont les mêmes ; mais dans un contexte, il y a une figure imposée par le marché (Barbie), alors que dans l'autre, on cherche un objectif pédagogique ponctuel (l'identification des lettres de l'alphabet).

Nous avons observé, dans une école, une proposition constituée par une petite boîte d'allumettes avec des billes. À travers une perforation faite sur la division interne en carton de la boîte, les billes pouvaient passer d'un côté à l'autre de la boîte, ce qui permettait l'apprentissage mathématique de l'addition et de la soustraction d'une dizaine. Ici, les habiletés des enfants, non seulement sont standardisées, mais aussi facilement mesurables. Le jeu est contrôlé. La question sous-jacente et que bien souvent nous nous sommes posée est : quelqu'un est en train de jouer? Ou, en d'autres termes, le sujet se manifeste-t-il ou est-ce la communitas qui le fait ? La maîtresse propose une action, qui se représente comme ludique per se ou ne le devient-elle dès lors qu'elle intervient dans la communauté de jeu?

La Loi Nationale de l'Éducation, sanctionnée en 2006 ( $\left.\mathrm{N}^{\circ} 26.206\right)$, parmi ses objectifs concernant le niveau de l'École Primaire (Maternelle et Élémentaire) préconise de: «Promouvoir le jeu comme une activité nécessaire pour le développement cognitif, affectif, éthique, esthétique, moteur et 
social» (chap II et III). Nous avons observé, dans notre recherche dans les écoles, que cette affirmation a été incorporée de diverses manières, mais suivant un axe commun : les jeux traditionnels comme une porte ouverte à la transmission de contenus didactiques d'une manière « implicite ».

Un enseignant, que nous nommerons Jean (36 ans), fait face à une double tension en ce qui concerne le jeu dans les premières années de l'école élémentaire. D'un côté, les élèves veulent jouer. D'un autre côté, l'utilisation du jeu comme ressource pédagogique suppose une grande tension face aux parents des élèves : «Mon fils ne vient pas ici pour jouer, c'est à la maison qu'il joue. Qu'il étudie.», a dit Marcela, la mère d'un élève de 7 ans, d'une école de la Ville de Buenos Aires (Cerletti 2014 et Santillán 2012).

Selon les enfants que nous avons interviewés (garçons et filles), le jeu s'oppose à l'étude et pour leurs parents également. Les enfants apprécient la présence des jeux en raison de leur dynamique. Ils reconnaissent qu'à l'école apparaissent des jeux «normaux, communs » que nous pourrions inclure dans la catégorie de "traditionnels ». Les adultes identifient l'espace familial comme l'espace de jeu, alors que l'école est pour eux un lieu d'apprentissage. Par conséquent le jeu est associé à une activité de loisir et non pas d'acquisition de connaissances. Leur préoccupation, concernant les enfants, porte sur la formation, l'apprentissage, peu importe de savoir quelle est la motivation qui explique que toute la classe joue aux échecs ou au jeu de dames; ou bien si l'on cherche la construction d'une pensée logique chez les enfants à travers l'utilisation de cartes numérotées. La perception des parents est que, pendant que l'on joue à l'école, on n'apprend rien.

Je ne sais pas très bien ce qu'ils font avec ces cartes-là. Fichez-moi la paix ! Qu'ils écrivent sur le cahier, qu'ils fassent des exercices de maths, je ne sais pas, moi, je ne comprends rien sur l'éducation! (Roberto, 30 ans, père de Romina et Mariano de 8 et 10 ans).

Quant au jeu, les contenus scolaires ont fait un virage important. En premier lieu, il a été inclus comme un support utile pour renforcer les contenus pédagogiques ou pour gérer des conflits entre collègues. Dans la conception des programmes en vigueur dans la ville de Buenos Aires (2004), le jeu apparaît lié à l'activité physique, à la musique, et aux expressions artistiques. Pourtant, nous n'y avons pas pu identifier d'une manière évidente la présence d'espaces de « jeu libre » (voir Duek et Enriz 2015 b).

Dans une série d'enquêtes que nous avons réalisées auprès d'enfants (garçons et filles) de 7 et 8 ans, nous leur avons posé des questions à propos des espaces de jeu dans l'école, en supposant que ce serait pendant la récréation que l'on identifierait les interactions les plus liées avec le fait ludique. Quelques interviewés ont affirmé: 
- On joue beaucoup dans la classe d'EPS (éducation physique et sportive).Le prof indique les règles et nous jouons tout le temps. C'est ce que j'aime le plus de l'école » (Tomás, 8 ans).

- La récré, c'est super, mais le mieux pour jouer c'est quand on est avec Miri en EPS et on court et elle nous laisse faire beaucoup de choses. Personne ne nous crie dessus, comme dans les récrés » (Marina, 7ans).

- Le mieux, c'est quand Mariano nous laisse jouer au football. Nous, les garçons, nous voulons jouer mais on ne nous laisse pas pendant la récré. Si on se comporte bien, une fois il nous laisse jouer, et les filles jouent au chat et à la souris dans la cour d'à côté » (Martín, 7 ans).

La principale nouveauté en ce qui concerne actuellement le jeu dans les écoles porte sur son intégration dans des programmes scolaires. Il existe une mise en scène de différents mécanismes persuasifs afin de présenter les activités masquées sous un aspect ludique. Que ce soit dans la classe d'EPS ou dans la salle de classe, l'apparition du jeu est en rapport avec des espaces réglés avec des objectifs et des zones délimitées, mais, pour les interviewés, il est identifié comme un temps-autre. Un temps avec des règles, certes, mais avec d'autres règles qui modifient le déroulement de la journée scolaire.

Les jeux traditionnels deviennent un support supplémentaire : il y a un cahier où l'on écrit, dans quelques cas, il y a des livres de texte pour travailler et aussi des espaces de jeu. On a toujours joué dans la salle de classe. Or le jeu, tel qu'il se manifeste de nos jours, prend une place mesurable lui permettant d'être analysé et évalué (Bally 1986 et Nunes 2013). Et c'est dans ce cadre que les appropriations et les transformations superficielles des jeux (qui continuent cependant à être pratiqués de la même manière) offrent une place privilégiée aux jeux traditionnels, ces pratiques étant aujourd'hui révisées, mais avec un rapport spécial avec le passé tout en incluant conceptuellement toutes les modifications subies à travers le temps.

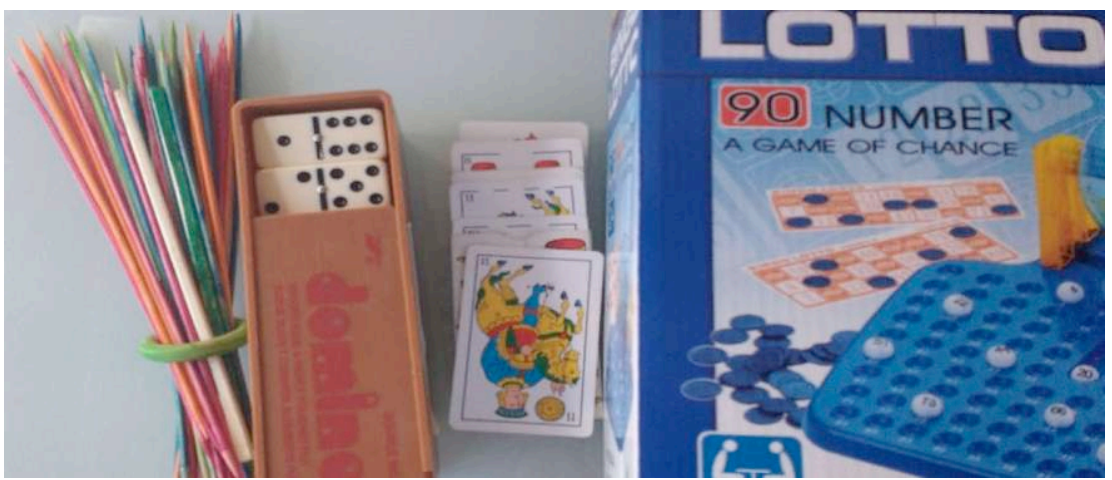

Image 2 : Mikado, domino, cartes et bingo (image des auteures) 


\section{La connexion, le foyer et les dispositifs}

Le temps vécu à la maison est différent de celui de l'école : les rythmes sont propres à chaque famille et les activités qu'on y réalise sont liées à la disponibilité, aux occupations et aux rôles de chacun à différents moments. Être « à la maison " c'est, pour plusieurs personnes, la possibilité de se détendre après une journée de travail et suppose l'occasion de faire une pause par rapport aux obligations. Les devoirs, les notes sur le cahier de liaison, les affaires qu'il faut apporter le lendemain (le cahier d'informatique, les outils pour Arts Plastiques, la boîte à lunch, pour en citer quelques exemples) ordonnent le temps familial.

Les enfants que nous avons interviewés à cette étape de notre recherche, nous ont reçues dans leurs chambres. Le but de ces interviews nétait pas seulement de parler avec eux mais aussi d'identifier les objets qui les entouraient quotidiennement. Plusieurs enfants dormaient dans le salon des maisons, d'autres dans des espaces improvisés divisés par des rideaux, mais la totalité avait un lit individuel qu'ils ne partageaient pas.

Pour eux, le temps du loisir est un temps consacré à la connexion numérique. Ils nous ont parlé des tablettes, des portables (personnels dans quelques cas, de leurs parents pour la plupart d'entre eux), d'ordinateurs et de consoles de jeux vidéo... La connexion numérique crée un temps ludique que les enfants opposent au temps scolaire. Alors qu'à l'école, la connexion est exceptionnelle, voire marginale, se forment au domicile, des « collectifs ludiques » autour de personnages de la télévision, des chanteurs, de YouTubers ou d'Instagramers. Ces « communautés de jeu ", ces « jeux de rôle » deviennent alors, pour les enfants, dans l'environnement familial, une opportunité de construction identitaire, un monde ludique commun très valorisé :

Je rentre chez moi, je prends le lait et je vais sur l'ordi. Maman arrive après moi, alors je lui dis que je viens de m'asseoir devant l'ordi quand elle entre. Je ne sais pas si elle me croit, mais elle me laisse y rester longtemps jusqu'à ce que je me baigne (Jonathan, 7 ans).

Je me connecte sur Internet chez moi quand je finis de manger à midi. Je m'amuse beaucoup et c'est super marrant (...) Je mennuie trop à l'école et ici je peux jouer avec l'ordi (Macarena, 8 ans).

Divertissement vs ennui; connexion vs non-connexion ; maison vs école : voici trois catégories que nous avons pu mettre en évidence à partir des témoignages. Or, le grand avantage méthodologique que nous fournit le fait d'interviewer les enfants chez eux, c'est de voir, comme nous l'avons déjà remarqué, les objets qui les entourent. Et c'est sur ce point que nous trouvons une différence entre ce qu'ils mentionnent et ce que nous avons observé. Tel que le soutient Bourdieu (1999), l'interview doit permettre un 
type de communication non violente tout en considérant, dès le début, que la recherche de la parole de l'autre s'avère être une intrusion.

Pour échanger avec les enfants, nous avons obtenu le consentement de leurs parents qui nous ont ouvert les portes de leurs maisons. Nous n'avons pas cherché, en aucun cas, à énoncer des "vérités» sur leur vie quotidienne mais plutôt la façon dont ils décidaient de la raconter face à un inconnu. Notre échantillon statistique (sur lequel s'appuie cet article), nous a permis de constater que les enfants interviewés possédaient au moins cinq objets ludiques clairement inclus dans la catégorie de « jeux traditionnels » : des cartes, des jeux de société, des ballons, des poupées, des élastiques et des autos. Visiblement, ces jeux dits « traditionnels » ne présentaient plus aucun intérêt pour eux. Face à nos questions, ils avaient des réponses de ce type:

Ah, oui, je ne joue pas beaucoup avec ça » ou « c'est plein de poussière, regarde, je n'utilise jamais ces petites autos-là.

Il est ressorti de notre enquête que les parents souhaitaient que leurs enfants renouent avec les jeux traditionnels : jeux de société, cartes, ballons, poupées... Dès lors nous avons relevé une autre dimension cruciale pour penser la place du jeu dans la sphère familiale: l'intervention des parents pour inciter les enfants à se "déconnecter». Autrement dit, l'utilisation excessive des objets numériques (dont la qualification du temps et les formes d'usage varient selon les familles en fonction de paramètres stipulés par leurs intégrants) incite les parents à exiger des enfants qu'ils en reviennent aux jeux traditionnels:

Si je le vois trop accro sur la Play (console de jeux vidéo),j'arrête un peu mon activité, je prends le domino et je l'invite à jouer. Il ne veut pas toujours jouer, mais quelquefois il dit que oui, au moins pour un moment il s'éloigne de la Play. C'est difficile, que dire! (María, 35 ans, mère d'Emiliano de 8 ans.)

Heureusement mon fils aime beaucoup jouer aux cartes, alors, si je lui dis d'y jouer, il vient et il joue. Mais, je n'ai pas toujours envie de jouer, je m’ennuie un peu. Pas pour lui, hein! C'est que j'arrive épuisée, le repas, le bain...Peut-être le weekend je massieds un peu plus, mais pendant la semaine je suis si crevée que je ne sais pas, je ne peux pas penser à m'asseoir pour jouer. (Sandra, 37 ans, mère de Robertino de 9 ans.)

Dans les cas analysés, l'apparition des jeux traditionnels est liée à l'intervention d'un adulte ou d'un frère qui les propose. Et lorsque l'adulte propose un jeu, de nouvelles dimensions d'analyse apparaissent :

Si on joue au bingo, au moins il fait une révision des chiffres jusqu'à 90 (Marcelo, père de Marianella de 9 ans).

Je lis parfois à Emma. On dit que la lecture aide les enfants à écrire mieux, n'est-ce pas? (Laura, 32 ans, mère d'Emma de 6 ans). 


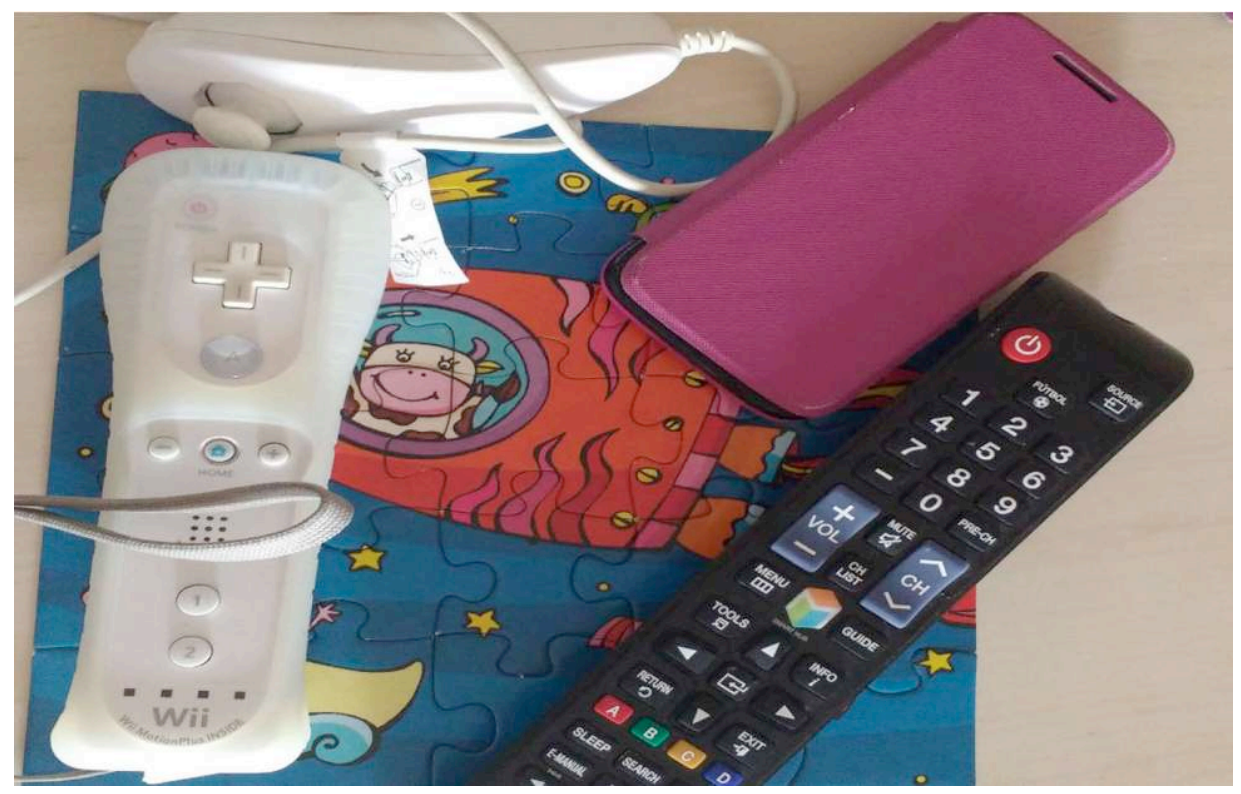

Image 3 : Puzzle, contrôles d'une console de jeux vidéo, portable et télécommande (photo des auteures)

L'utilité devient alors un horizon d'attente: il ne s'agit pas seulement de «déconnecter» les enfants des dispositifs mais de le faire d'une façon constructive. La préoccupation pour l'«utilité» reproduit l'inquiétude éducative préalablement mentionnée: à l'école, les enfants jouent pour apprendre, à la maison ils jouent pour apprendre aussi. Si c'est ainsi, quelle est la place pour le jeu libre, non orienté et sans utilité? Les communautés de jeu sont une réponse théorique et ethnographique possible pour réfléchir sur la place des jeux dans presque tous les domaines quotidiens des enfants. L'activité ludique, dans différents contextes, est vécue par les enfants comme une manière d'apprendre à faire monde commun.

\section{Réflexions finales}

On peut conclure, à partir des argumentations précédentes, que le jeu fait l'objet d'interprétations différentes selon les acteurs sociaux. Nous avons vu que les enseignants s'appuient sur les caractéristiques du jeu pour rapprocher leurs propres intérêts des contenus scolaires. Les parents retrouvent dans le jeu un espace où l'on " apprend ", tout en le différenciant des apprentissages scolaires « attendus ».Les enfants, de leur côté, perçoivent dans les propositions scolaires ludiques une modalité d'exigence éducative plus amusante et une possibilité récréative. Les marges du jeu admettent plus de flexibilité que les autres domaines institutionnels proposés. 
L'axe de notre argumentation a été centré sur les pratiques ludiques plutôt que sur le jeu. Nous avons trouvé en celles-ci la possibilité de penser au-delà des structures plus rigides : l'amusement peut bien commencer lorsqu'on distribue les fiches (ou les cartes) pour céder la place au jeu proprement dit. De même, la pratique ludique débute quand quelqu'un arrive en sautillant jusqu'au meuble où l'on garde les fiches, il prend la boîte, puis il simule qu'elles tombent, les jette, les ramasse, etc.

Dans ce texte nous avons considéré l'importance de la notion de communauté de jeu qui, bien que déjà utilisée dans la bibliographie spécifique pour les populations guaraníes (Enriz, 2011 ; Meliá 1968 ; Larriq, 1993), nous permet en même temps d'ouvrir le champ d'analyse sur cette catégorie et l'articuler avec des auteurs d'autres domaines, afin d'approfondir l'étude de l'incidence de la notion de communauté dans les contextes et les exemples déjà analysés.

Les Guaranies sont une population de 100000 personnes et vivent dans une région englobant une partie du Brésil, du Paraguay et de l'Argentine. Ils vivent dans des terres communales, ils parlent leur propre langue (qui a cinq variantes selon les zones d'utilisation) et pratiquent leur religion. La langue et la religion relèvent d'une transmission communautaire exclusive. Et c'est la raison pour laquelle elles deviennent centrales dans la conformation de la personne guarani dans le cadre d'une communauté qui la contient.

La communauté de jeu, en tant que notion, est construite ethnographiquement dans le but de rendre compte du rôle particulier que constitue le groupe d'enfants (garçons et filles) de différents âges qui, dans les communautés guaranies, est accompagné par la reconnaissance de leur communauté et de l'environnement naturel et socioculturel dans lequel ils vivent. Cet ensemble d'enfants, âgés de 2 à 11 ans, joue un rôle essentiel dans la formation des enfants des communautés, non seulement dans le développement de chacun d'eux comme membre du groupe, mais aussi comme le prélude de la communauté en tant que telle. D'ailleurs, dans le même sens proposé par Esposito (2007) pour d'autres communautés, la communauté de jeu excède et précède les sujets. C'est pour cette raison que nous avons repris ce concept pour réfléchir sur l'utilisation et les appropriations des jeux dans différents contextes ainsi que sur le regard des adultes par rapport à ces pratiques enfantines.

Cette expérience de communauté, telle qu'elle se construit dans chaque cas, est porteuse d'une hétérogénéité singulière, ce qui permet d'aborder des expériences sans manquer de multiplicité ni de métamorphose d'un sujet complexe (Gramaccia 2013). C'est ainsi que la métamorphose, qui se présente dans le processus complexe de la conformation et du support de la communauté, présuppose des transformations de sa temporalité, de son ampleur et de ses hiérarchies qui n'empêchent, pourtant, ni sa reproduction ni sa continuité. Il s'agit donc d'une communitas trascendante.

La possibilité que le jeu permette la construction de certains niveaux d'autonomie est liée au jeu libre, qui ne se définit pas par la liberté «absolue» 
des sujets, sinon par les marges de manœuvre, de négociation et les possibilités d'intervention que les enfants obtiennent à travers ou dans la pratique ludique. S'ils sont capables de jouer sans l'intervention des adultes, c'est parce que, comme communauté, ils ont acquis une certaine autonomie (temporaire). Et bien que cette autonomie puisse être liée aussi en partie aux désirs et aux projections de certains adultes, elle suppose quelque chose que les enfants capitaliseront pour eux. L'utilité prend alors un autre sens: il s'agit que les enfants puissent enrichir leurs propres expériences comme sujets sociaux en contact avec d'autres enfants à travers le jeu. Construire un monde imaginaire, habiliter de nouveaux liens avec l'entourage et jouer avec et à travers le monde qui entoure les enfants, peut se présenter sous une table, sur des chaises, à travers un jeu sur la toile on line ou sur un papier blanc. Il ne s'agit pas des objets mais des formes d'appropriation: c'est dans l'espace d'appropriation des jeux où nous retrouvons la recherche d'une utilité en tant que synonyme d'un résultat capitalisable. Le jeu permet la construction de communautés comme l'espace de dépassement du sujet. Jouer est construire constamment un lien avec le présent, avec le passé et avec un imaginaire sur l'avenir, dont la mise en scène est toujours la communauté.

\section{BIBLIOGRAPHIE}

AIZENCANG N., 2005, Jugar, aprender y enseñar. Relaciones que potencian los aprendizajes escolares, Buenos Aires: Manantial.

ALVAREZ-URÍA F. et VARELA J. (1991) Arqueología de la escuela, Madrid, La Piqueta.

BALY G., 1986, El juego como expresión de libertad, México, FCE.

BOURDIEU P., 1999, La miseria del mundo, Buenos Aires, FCE.

BROUGÈRE G., 2013, «E1 niño en la cultura lúdica» dans Revista Lúdicamente, n 4, Buenos Aires.

CALLOIS R., 1967, Los juegos y los hombres. La máscara y el vértigo, México, FCE.

CERLETTI L., 2014, Familias y escuelas, Buenos Aires, Biblos.

CHAPELA L., 2002, El juego en la escuela, México, Paidós.

DUEK C., 2012, El juego y los medios. Autitos, muñecas, televisión y consolas, Buenos Aires, Prometeo Libros.

DUEK C. et ENRIZ N., 2015a, Les jeux, les enfants et les nouvelles technologies : une relation culturelle et complexe dans Revue française des sciences de l'information et de la communication, $\mathrm{n}^{\circ}$ 6, France.

DUEK C. et ENRIZ N., 2015b, Juegos, interacción y sociabilidad. Vida cotidiana y representaciones sociales en y a través del juego dans Cuerpo, Educación, Política: tensiones epistémicas, históricas y prácticas, Galak, E. y Gambarotta, E. (comps.), Buenos Aires, Biblos. 
ENRIZ N., 2011, Jeroky Porã, Juegos Saberes y Experiencias infantiles Mbyá guarani en Misiones, LINCOM Studies in Anthropology, $\mathrm{n}^{\circ}$ 14, Allemagne.

ESPOSITO R., 2007, Communitas: origen y destino de la comunidad. Trad. Carlo Rodolfo Molinari Marotto, Buenos Aires, Amorrortu.

GEERTZ C., 1972, La interpretación de las culturas, Barcelona, Gedisa.

GRAMACCIA G., 2013, L'imaginaire du lien social dans les communautés électroniques, dans L'imaginaire et la représentation des nouvelles technologies de communication, Nicole Denoit (Dir), Tours, Presses Universitaires François Rabelais.

HABERMAS J., 1981, Historia y crítica de la opinión pública, Barcelona, Gustavo Gilli. HUIZINGA J., 1938, Homo Ludens, Buenos Aires, Emecé.

LARRICQ, M., 1993, Ipytŭ tŭ ma construcción de la persona entre los Mbya-guaraní, Universidad Nacional de Misiones, Editorial universitaria.

LÉVI-STRAUSS C., 1966, El pensamiento salvaje, Buenos Aires, Fondo de Cultura Económica.

MALAJOVICH A. et WINDLER R., 2000, Diseño curricular para la educación inicial. Marco general, Secretaría de Educación, Gobierno de la Ciudad de Buenos Aires.

MANSON M. et DE LA VILLE I., 2013, Los fabricantes de juguetes y juegos franceses y su responsabilidad social respecto del niño (1891-1911) dans Revista Lúdicamente, $\mathrm{n}^{\circ}$ 4, Buenos Aires.

MANTILLA L., 1996, «La clasificación de los juegos y su práctica regulada y vigilada en torno al género», dans Revista la Tarea, Guadalajara, México.

MELIÁ B., 1968, El guaraní conquistado y reducido, Asunción, Biblioteca paraguaya de antropología. Universidad Católica.

MILSTEIN D. et MENDES H., 1999, La escuela en el cuerpo. Estudios sobre el orden escolar y la construcción social de los alumnos e escuelas primarias, Buenos Aires, Miño y Davila.

NUNES A. 2013, Entre juegos y tareas : Una etnografía de las actividades cotidianas de los niños A'uwë-Xavante (MT, Brasil) en Revista Lúdicamente, n 4, Buenos Aires.

OROZCO GÓMEZ G. 1994 (coord), Televidencia. Perspectivas para el análisis de los procesos de recepción televisiva, México, Universidad Iberoamericana.

QUÉRÉ L., 2003, Le public comme forme et comme modalité d'expérience, CURRAPP, Le sens du public : Paris.

SANTILLÁN L., 2012, Quienes Educan a los chicos. Infancias trayectorias educativas y desigualdad, Buenos Aires, Biblos.

SENNETT R., 1978, El declive del hombre público, Barcelona, Ediciones Península.

WILLIAMS R., 1988, Marxismo y literatura, Barcelona, Península/Biblos.

Résumé : Dans ce texte, nous analyserons à la fois le lieu et l'appropriation du lieu des jeux par différents acteurs et espaces : la télévision, les écoles, les maisons, l'espace en ligne, les comportements autorisés et les interdits. Le jeu est considéré comme un espace au sein duquel les enfants produisent et reproduisent des aspects de la vie sociale encadrés dans les trajectoires culturelles, éducatives, économiques et sociales. Le thème 
central de cette proposition portera sur la nécessité de transformer le jeu en une pratique utile pour la conformation des communautés qui partagent des significations autour de leurs usages et appropriations des objets et de l'environnement. La communauté représente ce qui entoure les sujets et leur empêche d'être tels qu'ils sont puisqu'elle les précède et elle les excède (Espósito 2007). À l'aide de recherches empiriques faites dans la ville de Buenos Aires, nous allons traiter les résultats sur la relation entre les enfants, les contenus scolaires et les loisirs. Parmi les axes de cet article, nous avons identifié un certain niveau de conflit entre les jeux traditionnels et les jeux contemporains comme deux domaines, à la fois source de dialogues et de conflits. Sur le plan méthodologique, le texte est le résultat d'entretiens avec des enfants et des adultes, des observations dans des établissements d'enseignement systématisés par les auteurs de ce document. Cette recherche nous permet de réfléchir sur le jeu, les joueurs et les crédits des discours sociaux. Mot-clés : jeu, enfants, appropriations.

Abstract: In this text we will analyze the place and the appropriations of play made by different actors and spaces: television, schools, homes and on-line space, the allowed behaviors and limited ones. Play is considered as a space in which children produce and reproduce varied aspects of their lives framed in their cultural, educational, economic and social trajectories. The main subject of this article is related to the need to transform play into a useful practice as it was identified along the research in the conformation of communities that share meanings around their uses and appropriations of objects and environment. The community represents what surrounds the subjects and prevents them from being as they are because it precedes them and exceeds (Espósito, 2007). Using data from inquiries made in the city of Buenos Aires, we will analyze the relationship between children, school content and leisure. Between the axes of the article we identified a certain tension and dialogue between traditional and contemporary games. Methodologically, this article is the result of interviews with children and adults, of observations in different schools, that were both produced and analyzed by us. The research that organizes the analysis allows us to reflect upon play, players and the social discourses around them.

Keywords: Play, Children, Appropriations.

Resumo: Este texto analisa ao mesmo tempo o relacionamento que diferentes atores e espaços desempenham com o jogo: televisão, escolas, casas, espaço online, comportamentos e proibiçôes autorizadas. O jogo é considerado como uma esfera sem qualidades onde as crianças produzem e reproduzem a vida social, cultural, educacional e econômica. O tópico central que abordamos é a necessidade de transformar o jogo em uma prática útil. Com base em uma pesquisa empirica realizada na cidade de Buenos Aires, tentaremos abordar os resultados de uma relação entre crianças, currículo e práticas. Entre os eixos deste artigo, identificamos um certo nivel de conflito entre jogos tradicionais e jogos contemporâneos, como dois dominios que têm diálogos e pontos de contato. No nivel metodológico, o texto é o resultado de entrevistas com crianças e adultos e observações em instituiçóes educacionais sistematizadas pelos autores neste documento. A partir da pesquisa básica, buscamos abordar as relações entre peça, jogadores e discurso social.

Palavras-chave: Jogo, crianças, apropriação. 